\title{
Factores asociados a la calidad de vida en escolares de San Juan de Lurigancho
}

\section{Factors associated with elementary school students' quality of life in San Juan de Lurigancho}

\author{
Danitsa Alarcón Parco*, Patricia Bárrig Jó** \\ Escuela Profesional de Psicología, \\ Universidad de San Martín de Porres, Perú
}

\section{Resumen}

Es importante conocer las variables que influyen en la calidad de vida en la niñez por ser esta un indicador del estado de salud física y mental futuros. En tal sentido, la presente investigación busca describir los factores asociados a la calidad de vida en una muestra de 400 escolares de instituciones educativas del distrito de San Juan de Lurigancho. Se desarrollan los factores asociados o que intervienen en la calidad de vida, tales como la composición familiar, el nivel socioeconómico, el estado civil, la ocupación de los cuidadores, la edad y el grado de instrucción de los padres. La investigación es de tipo descriptivo correlacional, se aplicaron dos cuestionarios para medir la calidad de vida y los factores psicosociales. Los resultados muestran niveles bajos en bienestar físico y se hallaron seis factores psicosociales asociados con la calidad de vida respecto al bienestar emocional, a la familia, a los amigos y al colegio.

Palabras clave: factores psicosociales, calidad de vida, niños, colegios de San Juan de Lurigancho. 


\begin{abstract}
It is important to know the variables that affect the quality of life in childhood, as it is a key indicator of both physical and mental health. In this regard, this study aims to describe the factors associated with the quality of life in a sample of 400 elementary students from schools in the district of San Juan de Lurigancho. Such factors associated with or affecting the quality of life are family structure, socioeconomic level, marital status, caregivers' jobs, and parents' age and education level. The research has a descriptive correlational design. Two questionnaires were used to measure the quality of life and psychosocial factors. The results showed low levels of physical well-being. Six psychosocial factors associated with quality of life regarding emotional well-being, family, friends and school were found.
\end{abstract}

Keywords: psychosocial factors, quality of life, children, schools in San Juan de Lurigancho.

En los últimos años, la calidad de vida viene cobrando importancia en la salud pública, aumentando su relevancia progresivamente en los diferentes ámbitos del quehacer profesional y científico. En el ámbito de la salud, los avances de la medicina han hecho posible prolongar la vida de las personas que adolecen enfermedades crónicas, lo cual ha repercutido en orientar la atención hacia la calidad de vida relacionada con la salud (Urzúa, 2010).

Para evaluar la calidad de vida se debe dar una mirada multidimensional que incluya aspectos como el estilo de vida, satisfacción familiar, escolar y laboral, así como el aspecto económico. La calidad de vida, entonces, es un sistema de valores que cambian de acuerdo a la persona, grupo y/o ciudad; es decir, es la sensación de bienestar que pueden experimentar las personas, manifestándose en sensaciones subjetivas y propias de cada persona. Rodríguez, Esteve y López (2000) indican que la calidad de vida es un análisis subjetivo que las personas realizan acerca de la felicidad, manifestado en sus sentimientos de bienestar o malestar, relacionados a indicadores biológicos, psicológicos, conductuales y sociales, donde encontramos la comunidad, la familia y el colegio. 
La conceptualización de la calidad de vida es una operación compleja. Los profesionales en salud tienden a subestimar el estado funcional y algunos síntomas físicos y a valorar algunas manifestaciones psicológicas. La calidad de vida se vincula a factores relacionados con el plan de vida del ser humano, formando así las condiciones de vida del individuo junto a la satisfacción personal. Sin embargo, estas condiciones de vida estarían excluyendo aspectos que intervienen directamente con la forma de interpretar las situaciones que se presentan, e influyen en el contexto sociocultural.

Teniendo en cuenta la influencia de la cultura es preciso mencionar la importancia de factores asociados o que intervienen en la calidad de vida, tales como la composición familiar, el nivel socioeconómico, el estado civil, la ocupación de los cuidadores, la edad y el grado de instrucción de los padres. Todo ello, variaría de acuerdo al desarrollo evolutivo, es decir, que la percepción estaría influida por factores ligados a la edad. A pesar de ello, la calidad de vida durante la niñez temprana ha recibido menor atención que en la adultez, por lo que se desconoce los factores asociados que acompañan al bienestar de niños y niñas, dejando de lado que una intervención o prevención temprana puede ayudar a mejorar este aspecto (Rajmil et al., 2001).

Como se ha mencionado, la calidad de vida, es importante en la vida del ser humano, ya que se plasma las experiencias satisfactorias que las personas tienen acerca de su condición de vida, siendo interpretadas de manera positiva o negativa, favoreciendo o afectando la relación con el medio o consigo mismo. En el caso de los niños, la calidad de vida es reportada por sus padres y/o tutores que se encargan de la crianza y están más pendientes de las necesidades de los hijos e hijas. Sin embargo, es importante contar con la percepción que tienen los niños acerca de sus propias necesidades personales, cómo se sienten consigo mismos, qué es lo que piensan de ellos, así como conocer cuál es la apreciación que tienen sobre el apoyo de la familia, las dinámicas familiares, los amigos, si le es fácil o no la convivencia con ellos, qué opinión tienen acerca de sus tareas, las clases, los profesores, o cómo perciben el colegio, ya que todo ello forma parte del contexto social en el cual viven y crecen. 


\section{Método}

\section{Participantes}

La muestra estuvo conformada por 400 niños de 8 a 12 años pertenecientes a seis instituciones educativas del distrito de San Juan de Lurigancho, seleccionados mediante un muestreo no probabilístico de tipo intencional (Hernández, Fernández, \& Sampiere, 2014), utilizando la fórmula para muestras finitas, con un nivel de confianza al $95 \%$ y un error muestral de 5\%. El 87.32\% compuesto por escolares de instituciones educativas estatales y $12.68 \%$ de instituciones privadas. Los niños de 8 y 9 años representan un $67.8 \%$ de la muestra y los niños de 10, 11 y 12 años $32.2 \%$. Siendo de sexo femenino el $52.3 \%$.

La mayoría de los participantes de la muestra nacieron por parto normal (77.3\%); no tuvieron problemas al nacer (81.8\%). El 60.3\% de la muestra indicó que los ingresos familiares permitían cubrir sus necesidades básicas, mientras que $6.5 \%$ reportó que solo les permitía cubrir alimentación. La mayoría reportaron tener padres que estaban casados (47.5\%); 33\% convivientes, y $17.8 \%$ tenían padres separados o divorciados. En cuanto a su ocupación, $54.3 \%$ correspondió a las amas de casa; 20.8\% a comerciantes $(20.8 \%)$, y $7 \%$ a empleadas en una organización. El 31.8\% de los padres se dedicaban al comercio; $30.5 \%$ eran obreros, y $21 \%$ empleados en una organización.

La mayoría de los niños de la muestra vivían con ambos padres (52.5\%), otro grupo vivía con ambos padres, hermanos y parientes $(29.8 \%)$ y finalmente se encuentran los que vivían con la madre o el padre, hermanos y parientes (11.8\%). Así mismo, un $68 \%$ de los niños y niñas están satisfechos con el tiempo que pasan junto a su familia; 20.5\% señalaron que a veces se encuentran satisfechos; y finalmente, $11.5 \%$ reportaron que no se encuentran satisfechos con su tiempo en familia.

Cuando necesitan atención médica se observa que los miembros de su familia son llevados al centro de salud (42\%), a un hospital $(31 \%)$ y a un policlínico (17\%). Para ellos, la calidad de la atención en los lugares que acuden es regular (49.8\%), buena (47.3\%) o mala (3\%). Por último, se detalla las características de acuerdo al material de construcción de su 
vivienda: $85.8 \%$ manifestó que su casa era de material noble y contaban con agua; $9 \%$ indicó vivienda de madera con servicios de agua y desagüe; y 5.3\% reportó vivir en casa de otro tipo de material y otro tipo de servicio.

\section{Diseño de investigación e instrumentos}

Se utilizó un diseño de tipo correlacional, no experimental y transversal. (Hernández et al., 2014; Kerlinger, 2004).

- Cuestionario de Calidad de vida para niños (KINDL KID). E1 Cuestionario de Calidad de Vida Relacionado con la Salud (CVRS) para Niños y Adolescentes (Kindl Kid) fue desarrollado en Alemania para ser utilizado en la práctica clínica, así como en niños sanos (Rajmil et al., 2004). Dicho cuestionario contiene 24 ítems distribuidos en seis dimensiones: Bienestar físico, Bienestar emocional, Autoestima, Familia, Amigos y Colegio. Cuenta con versiones para cada grupo de edad: Kiddy-Kindl, para los niños de 4-7 años, Kid-Kindl de 8-12 años, y Kiddo-Kindl de 13-16 años. Las dos primeras con versiones para los padres o tutores. Los ítems son afirmaciones que deben ser respondidas respecto a lo sucedido a la semana previa a la evaluación y las puntuaciones de cada dimensión se transforman a una escala de 0 a 100 puntos, siendo una mayor puntuación la que representa mejor Calidad de Vida Relacionada a la Salud (CVRS). Las respuestas del Kindl kid son cinco opciones de la escala Likert. La puntuación total o índice global de Calidad de Vida Relacionada a la Salud (CVRS) se obtiene a partir de las medias de las seis dimensiones.

La consistencia interna fue superior a .70 en el puntaje total de CVRS y en tres de las seis dimensiones del Kindl, con excepción de las dimensiones de bienestar físico, amigos y colegio. La estabilidad test-retest presentó CCI mayor a .60 menos las dimensiones de bienestar físico y bienestar emocional. Es importante mencionar que resultaron más bajos en los más pequeños (8-11 años). La versión española y la versión original alemana, presentaron similares resultados de fiabilidad y validez. 
- Cuestionario de los Factores Psicosociales. Se diseñó un cuestionario para conocer los factores asociados a la calidad de vida, dividida en factores psicosociales: edad, sexo, nivel educativo, colegio (nacional o particular), número de hermanos y el lugar que ocupa, nivel socioeconómico, lugar de procedencia de los padres, estado civil de los padres, grado de instrucción de los padres, ocupación de los padres, composición familiar, edad de los padres.

\section{Procedimiento}

Se aplicó la prueba de ajuste K-S a la muestra para determinar si esta, presenta una distribución normal o no, y así establecer el tipo de estadístico a utilizar. Con la finalidad de determinar los factores psicosociales asociados con la calidad de vida, se dividió el análisis en dos partes: en la primera parte se analizaron los factores psicosociales de naturaleza cuantitativa y en la segunda parte se analizaron los factores psicosociales de naturaleza cualitativa.

Para el análisis cuantitativo, en primer lugar, se verificó si los datos tenían una distribución normal, encontrándose que los factores psicosociales de naturaleza cuantitativa, así como las subescalas de calidad de vida y el puntaje total de esta variable no se aproximan a una distribución normal, por lo tanto, se analizaron con una prueba no paramétrica, específicamente la prueba de correlación de Spearman.

La prueba no paramétrica del chi cuadrado de Pearson (Greene \& D'Oliveira, 2006) establece asociaciones con las variables y las asociaciones aceptables se trabajan con un nivel de significación estadística de .05 para rechazar o aceptar la hipótesis de investigación. Se utilizó también la $t$ de Student, para comparaciones entre dos condiciones (Greene \& D'Oliveira, 2006), permitiendo así conocer la media de dos grupos que difieren entre sí con un nivel de significancia estadística .01 para rechazar o aceptar la hipótesis de investigación. Para comparaciones de promedios de las variables estudiadas en función de los grupos etarios (edades) se utilizó la técnica de Análisis de Varianza (ANOVA). 


\section{Resultados}

En la Tabla 1 se observa el puntaje donde los niños y niñas de San Juan de Lurigancho presentan un bajo nivel de bienestar físico y calidad de vida relacionado al colegio, mientras que $44.3 \%$ presentan un bienestar emocional promedio respecto a los demás. Sin embargo, $48 \%$ presenta una calidad de vida general en nivel promedio. Los niveles altos de la calidad de vida están en relación con la familia y con alguna enfermedad.

Tabla 1

Niveles de calidad de vida en general y sus dimensiones

\begin{tabular}{|c|c|c|c|c|c|c|c|c|}
\hline Niveles & $\begin{array}{l}\text { Bienestar } \\
\text { físico }\end{array}$ & $\begin{array}{l}\text { Bienestar } \\
\text { emocional }\end{array}$ & $\begin{array}{c}\text { Relacionada } \\
\text { a la } \\
\text { autoestima }\end{array}$ & $\begin{array}{c}\text { Relacionada } \\
\text { con la } \\
\text { familia }\end{array}$ & $\begin{array}{l}\text { Relacionada } \\
\text { al colegio }\end{array}$ & $\begin{array}{l}\text { Relacionada } \\
\text { a los } \\
\text { amigos }\end{array}$ & $\begin{array}{l}\text { Relacionada } \\
\text { a alguna } \\
\text { enfermedad }\end{array}$ & $\begin{array}{l}\text { Calidad } \\
\text { de vida } \\
\text { general }\end{array}$ \\
\hline Bajo & 41.5 & 27.8 & 37.3 & 37.8 & 40.8 & 35.8 & 30.0 & 25.5 \\
\hline Promedio & 32.5 & 44.3 & 30.3 & 12.3 & 33.0 & 32.0 & 19.0 & 48.0 \\
\hline Bueno & 26.0 & 28.0 & 32.5 & 50.0 & 26.3 & 32.3 & 51.0 & 26.5 \\
\hline
\end{tabular}

Nota: Las cifras presentadas se encuentran en porcentaje, teniendo en cuenta cuartiles: $\leq 25$ Calidad de vida baja, $\geq 75$ Calidad de vida bueno.

\section{Identificación de factores asociados}

Entre los factores psicosociales asociados a la calidad de vida se encontró que el número de hermanos se relaciona de manera negativa con el bienestar físico $(r=-.14, p<.01)$, con colegio $(r=-.12, p<.05)$ con enfermedad $(r=-.12, p<.05)$ y con calidad de vida en general $(r=-.14, p<.01)$; es decir, que a mayor número de hermanos, existe menor calidad de vida relacionada con el bienestar físico, el colegio, la enfermedad y la calidad de vida en general. Cabe mencionar que estas correlaciones son débiles. Por su parte, el tiempo de residencia de la madre en Lima se relaciona de manera directa con la calidad de vida con respecto a la enfermedad $(r=.13$, $p<.05)$, lo que indica que a mayor tiempo de residencia de la madre en Lima, se presenta una mayor calidad de vida relacionada con la enfermedad; empero, esta relación también es débil. En cuanto al tiempo de residencia del padre en Lima, se relaciona de manera directa y débil con el bienestar emocional $(r=.10, p<.05)$ y con la calidad de vida en general $(r=.10, p$ $<.05)$. 
En cuanto, a la edad de la madre se encontró una relación directa con el bienestar emocional $(r=.11, p<.05)$ y con la calidad de vida en general $(r=.12, p<.05)$; es decir, que a mayor edad de la madre, existe mayor calidad de vida relacionada con el bienestar emocional y con la calidad de vida en general. En relación a la edad del padre, esta se relaciona de manera directa con la autoestima $(r=.11, p<.05)$ y con la calidad de vida en general $(r=.11, p<.05)$; es decir, que a mayor edad del padre, existe mayor calidad de vida relacionada con la autoestima y con la calidad de vida en general.

Otro factor psicosocial asociado a la calidad de vida respecto al bienestar físico es el lugar de nacimiento de la madre $\left(\chi_{(\mathrm{n})}^{2}=13.37, p=.010\right)$. Factores asociados con la calidad de vida respecto al bienestar emocional, se encontró al nivel educativo $\left(\chi_{(\mathrm{n})}^{2}=13.25, p=.039\right)$, nivel socioeconómico $\left(\chi_{(\mathrm{n})}^{2}=18.13\right.$, $p=.020)$, calidad de atención que recibe $\left(\chi_{(\mathrm{n})}^{2}=15.33, p=.004\right)$, ingresos familiares $\left(\chi_{(\mathrm{n})}^{2}=19.07, p=.004\right)$ y el material de construcción de la casa $\left(\chi_{(\mathrm{n})}^{2}=11.62, p=.020\right)$.

Se encontraron cinco factores psicosociales asociados con la calidad de vida respecto al bienestar emocional. Estos factores fueron: parto $\left(\chi_{(\mathrm{n})}^{2}=\right.$ 7.53, $p=.023)$, lugar de nacimiento de la madre $\left(\chi_{(\mathrm{n})}^{2}=10.16, p=.038\right)$, ocupación del padre $\left(\chi_{(\mathrm{n})}^{2}=36.61, p=.001\right)$, satisfacción con el tiempo que pasa en familia $\left(\chi_{(\mathrm{n})}^{2}=16.39, p=.003\right)$ y los ingresos $\left(\chi_{(\mathrm{n})}^{2}=13.45, p=.036\right)$.

Además, se encontraron seis factores psicosociales asociados con la calidad de vida respecto a la familia. Estos factores fueron: nivel educativo $\left(\chi_{(\mathrm{n})}^{2}=17.63, p=.007\right)$, colegio $\left(\chi_{(\mathrm{n})}^{2}=13.62, p=.001\right)$, lugar que ocupa $\left(\chi_{(\mathrm{n})}^{2}=31.57, p=.005\right)$, ocupación de la madre $\left(\chi_{(\mathrm{n})}^{2}=32.60, p=.003\right)$, situación laboral del padre $\left(\chi_{(\mathrm{n})}^{2}=14.00, p=.007\right)$ y satisfacción con el tiempo que pasa en familia $\left(\chi_{(\mathrm{n})}^{2}=31.13, p=.000\right)$.

En cuanto a los factores psicosociales asociados con la calidad de vida respecto a los amigos, se encontraron siete asociaciones: nivel educativo $\left(\chi_{(\mathrm{n})}^{2}\right.$ $=23.55, p=.001)$, colegio $\left(\chi_{(\mathrm{n})}^{2}=7.09, p=.029\right)$, parto $\left(\chi_{(\mathrm{n})}^{2}=6.19, p=\right.$ $.045)$, ocupación de la madre $\left(\chi_{(\mathrm{n})}^{2}=25.36, p=.031\right)$, personas con quien vive $\left(\chi_{(\mathrm{n})}^{2}=12.70, p=.48\right)$, satisfacción con el tiempo que pasa en familia $\left(\chi_{(\mathrm{n})}^{2}=22.79, p=.000\right)$ y los ingresos $\left(\chi_{(\mathrm{n})}^{2}=13.13, p=.041\right)$. 
Tres factores psicosociales asociados con la calidad de vida respecto al colegio fueron nivel educativo $\left(\chi_{(\mathrm{n})}^{2}=19.20, p=.004\right)$, calidad de atención que recibe $\left(\chi_{(\mathrm{n})}^{2}=12.77, p=.012\right) \mathrm{y}$ los ingresos $\left(\chi_{(\mathrm{n})}^{2}=15.73, p=.015\right)$.

Tres factores psicosociales se encontraron asociados con la calidad de vida respecto a la enfermedad. Estos factores fueron: nivel socioeconómico $\left(\chi_{(\mathrm{n})}^{2}=21.09, p=.007\right)$, ocupación de la madre $\left(\chi_{(\mathrm{n})}^{2}=26.55, p=.022\right) \mathrm{y}$ ocupación del padre $\left(\chi_{(\mathrm{n})}^{2}=40.56, p=.000\right)$.

Finalmente, tres factores psicosociales fueron asociados con la calidad de vida. Estos factores fueron: parto $\left(\chi_{(\mathrm{n})}^{2}=7.79, p=.020\right)$, satisfacción con el tiempo en familia $\left(\chi_{(\mathrm{n})}^{2}=14.37, p=.006\right)$ y material de construcción de la $\operatorname{casa}\left(\chi_{(\mathrm{n})}^{2}=16.64, p=.002\right)$.

\section{Discusión}

Los factores psicosociales asociados a la calidad de vida, como el parto, la ubicación en el orden de nacimiento y el nivel socioeconómico coinciden con otras investigaciones que reportaron la relación significativa y positiva entre nivel socioeconómico y calidad de vida (Juarez et al., 2002; Sabeh et al., 2004; Solano et al., 2017; Woods \& Honig, 2006). Sin embargo, no hay reportes que evidencien la relación entre el lugar de nacimiento de los padres, el tiempo de residencia de los padres y la calidad de vida respecto a la enfermedad, solo la nuestra. Empero, estado civil de los padres y la ocupación de ellos se relaciona de manera directa con la calidad de vida de los niños, y coincide con el estudio en una población española que encuentra diferencias en la calidad de vida infantil en función de la satisfacción familiar, estructura familiar o estado civil, entendiéndose este con la presencia de ambos padres o de uno de los padres y/o si han formalizado su relación o se han divorciado/separado, por el impacto que tiene el divorcio en la calidad de vida de los niños (Harker, 2006; Orgilés \& Samper, 2011; Solano et al., 2017).

Sí se habla de las diferencias en la calidad de vida, respecto al género, a la edad, a la situación laboral de los padres y al nivel educativo, ya que está relacionado a lo que cada padre pueda ofrecer al niño como seguridad o recreación, como se ha reportado en otras investigaciones, tanto 
psicométricas, cuantitativas y cualitativas (Harker, 2006; Solano et al., 2017; Verdugo \& Sabeh, 2002).

La mayoría de las investigaciones coinciden -al referirse a la calidad de vida relacionada a la salud- en darle importancia a la calidad de atención de la enfermedad, tipo y tiempo de enfermedad y el alcance económico de los servicios de salud, ya que contribuyen a la calidad de vida de manera favorable, otorgando al niño bienestar emocional y psicológico (Danckaerts et al., 2010; Harker, 2006; Rodríguez, Bastidas, Genta, \& Olaya-Contreras, 2016; Solano et al., 2017; Wetzman et al., 2013).

Cabe indicar que los trabajos de investigación presentados en los antecedentes están referidos a la variable calidad de vida en pacientes hospitalizados o a pacientes con determinada enfermedad, a diferencia del presente trabajo, que se refiere a cómo los estudiantes del nivel de educación primaria, de dos instituciones educativas, perciben la calidad de vida (Gonzáles, 2004; Juárez et al., 2002).

Desde el punto de vista teórico, la existencia de una serie de factores de riesgo que determinan la calidad de vida, siempre van a estar delimitados por las variables sociales y demográficas que condicionan el estilo de vida o los hábitos que tienen los miembros de una comunidad en particular.

Cuando se habla de calidad de vida, se refiere a un proceso fenomenológico a partir de cómo los individuos perciben el impacto de los factores sociodemográficos que afectan la calidad de vida. Es importante, tener en cuenta que la calidad de vida, como un proceso subjetivo, es relativa y cambiante en función del estado orgánico y psicológico de los individuos evaluados.

Los resultados de esta investigación sirven de base para diseñar programas de prevención en las instituciones educativas de la red de San Juan de Lurigancho, con el fin de trabajar el tema de calidad de vida y factores asociados. Asimismo, se puede capacitar a los cuidadores a través de las escuelas de padres; también a los docentes para que orienten y asesoren a los estudiantes respecto a su calidad de vida. Es importante realizar un trabajo multidisciplinario tanto en niños como adultos. 


\section{Referencias}

Danckckaerts, M., Sonuga-Barke, E., Banaschewski, T., Buitelaar, J., Döpfner, M., Hollis, C., \& Coghill, D. (2010). The quality of life of children with attention deficit/hyperactivity disorder: a systematic review. European Child \& Adolescent Psychiatry, 19(2), 83-105.

Gonzáles, F. (2004). Calidad de vida en niños hospitalizados de Castilla y León (Tesis doctoral). Universidad de Salamanca, España.

Greene, J., \& D'Oliveira, M. (2006). Test estadísticos para psicología (3ª ed.). España: McGraw-Hill.

Harker, L. (2006). Chance of lifetime the impact of bad housing on children's lives. Reino Unido: Shelter.

Hernández, R., Fernández, C., \& Sampiere, P. (2014). Metodología de la investigación. México: McGrawHill.

Juárez, J., Torres, L., Osorio, M., Dueñas, T., Marín, T., Ortiz, A., ..., Rozen-Fuller, E. (2002). Calidad de vida del niño leucémico en fase terminal. Boletín Médico del Hospital Infantil de México, 59(7), 403-411.

Kerlinger, F. N. (2004). Enfoque conceptual de la investigación del comportamiento. México: Nueva Editorial Interamericana.

Rajmil, L., Serra-Sutton, V., Fernandez-Lopez, J., Berra, S., Aymerich, M., Cieza, A., ..., Ravens-Sieberer, U. (2004). Versión española del cuestionario alemán de calidad de vida relacionada con la salud en población infantil y de adolescentes: el Kindl. Anales de Pediatría, 60(6), 514-521.

Rodríguez, J., Bastidas, M., Genta, G., \& Olaya-Contreras, P. (2016). Calidad de vida relacionada con la salud en escolares de 10 a 14 años con sobrepeso y obesidad en la ciudad de Medellín, Colombia. Universitas Psychological, 15(2), 301-314.

Rodríguez, J., Esteve, R., \& López, E. (2000). Dolor crónico y estrategia de afrontamiento. Análisis y Modificación de la Conducta, 26(107), 391-418.

Solano, F., Vilela-Estrada, M., Meza-Liviapoma, J., Araujo-Chumaceroa, M., Vilela-Estrada, A., \& Mejía, C. (2017). Factores sociofamiliares asociados a la calidad de vida en niños de colegios de la ciudad de Piura, Perú. Revista Chilena de Pediatría, 88(2), 223-229.

Urzúa, A. (2010). Calidad de vida relacionada con la salud: elementos conceptuales. Revista Médica de Chile, 138, 341-348.

Verdugo, M., \& Sabeh, E. (2002). Evaluación de la percepción de calidad de vida en la infancia. Psicothema, 14(1), 86-91.

Wetzman, M., Baten, A., Rosentahl, D., Hoshino, R., Tohn, E., \& Jacobs, D. (2013). Housing and child health. Current Problem Pediatric Adolescent Health Care, 43(8), 187-224. 